\title{
Tadataka Yamada
}

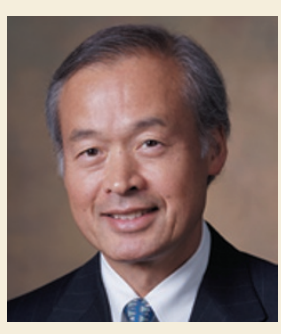

President, Global Health Program, Bill \& Melinda Gates Foundation, Seattle, USA. Tadataka (Tachi) Yamada is President of the Global Health Program at the Bill \& Melinda Gates Foundation. As president, Yamada leads the foundation's efforts to help develop and deliver low-cost, life-saving health tools for the developing world. Before joining the foundation in 2006, Yamada was Chairman of Research and Development and a member of the Board of Directors at GlaxoSmithKline (GSK). Earlier this year, he was made an honorary Knight Commander of the Order of the British Empire (KBE) in recognition of his contributions at GSK.

Could you give examples of successes that the Gates Foundation has had in promoting the discovery and development of drugs for neglected diseases?

At this point we have one drug called paromomycin that has been approved for registration in India. Paromomycin is an old aminoglycoside that the Institute for OneWorld Health identified for its potential to treat visceral leishmaniasis. The Gates Foundation completely sponsored the drug through clinical development and we are about to launch it through a network of private providers called Janani.

From the Medicines for Malaria Venture there are four compounds in Phase III and we sponsored a GSK malaria vaccine called RTSS that will enter Phase III in 2008. Also, we have six vaccine candidates about to start Phase II trials for Mycobacterium tuberculosis infection. Our portfolio has more than 70 new chemical entities or vaccines near clinical development.

\section{What lessons have been learnt in drug} discovery for neglected diseases over the past 7 years?

One key issue is the capacity for doing clinical trials in the developing world. For example, when conducting HIV prevention trials it is hard to find populations that are at a high enough risk for HIV so it becomes difficult to enrol enough patients. If you have many trials at the same time you easily exhaust the clinical trials capacity, which is what happened with our microbicide studies. We had four or five first generation microbicide studies at the same time, which made it difficult for us to start our second generation trials. Also, when you conduct HIV prevention trials you have to implement a comprehensive education strategy on the standard of care interventions against HIV, which means that the underlying incidence drops in the control group as well as in the test group. More patients are then needed to determine if the prevention product works.

Another important lesson is that although we have made large investments, not all are going to pay off. It is important to recognize that failure is more common than success.

How does the Gates Foundation engage industry to encourage the development of drugs for neglected diseases?

When we enter into a product development partnership with a pharmaceutical company we tend to support the research and development of the drug while the company retains its intellectual property. In return, we only ask for an affordable price for markets in the developing world.

Aside from our product development partnerships we have created funding mechanisms to ensure that industry is not penalized for participating in this area. One example is the Advanced Market Commitment directed at rewarding companies following success so their capital investment is reimbursed appropriately. Earlier this year we made our first advanced market commitment of $\$ 1.5$ billion for a pneumococcal vaccine.

\section{Early in October the Gates Foundation} announced a new fast-track grants programme to support innovative global health research. What are the main aims of this initiative and why is it needed?

First of all, we want to encourage novel ideas from anywhere in the world - Bill Gates often tells me that almost all technology innovation comes from China or India. However, in biomedical research most funding vehicles are in the United States or Western Europe. Second, we want a faster response to innovative thinking - traditional funding processes often give grants one or two years after the initial application. We also believe that peer review, although important, tends to stifle innovation because truly novel ideas have no peers. So the idea is to provide US $\$ 100,000$ grants within 3 months of submission of a two-page application that does not require preliminary data. We hope the grant reviewers will be creative people, reasonably versed in the sciences, who have demonstrated an ability to think about problems in a unique way.

We need this scheme because, if you look at a challenge like HIV, 25 years after the emergence of the disease we do not have a vaccine. I think we are mired in some old ideas and we need novel ideas to solve this problem.

From your experiences at GSK, what do you think are the major challenges for pharmaceutical companies to engage in addressing long-neglected health problems? Traditionally these efforts have been looked on as costly, owing to the financial investment and the cost of applying people and useful resources against products that will not be profitable. From the shareholder perspective that might not be optimal. However, pharmaceutical companies have an important challenge to be committed to the people who are suffering across the world, whether or not they can make money from them. Over time there has been a tremendous loss of esteem for the industry which has translated to pressures on pricing, market access and on regulatory approval. By contributing meaningfully to the broad population of people who need help, the cost will be small measured against the returns in reputation, corporate self esteem and general satisfaction of employees. From the experience at GSK, helping address these problems gave the company a sense of pride and motivation - that positive impact is almost incalculable.

This is a unique time as nations, corporations, foundations and the general public have focused on global health, which has led to new sources of funding. Most importantly, science has advanced to the stage that solutions to difficult problems are possible with this coalescence of forces. It is imperative that the pharmaceutical industry be a partner in this. We need them, we value them and we want to look for ways that they can be rewarded for what they do. Their reward in return will be that they can help millions of people. 